\title{
Begonia socotrana, die Weihnachts-Begonie
}

\author{
Annette Höggemeier \&Veit Martin Dörken
}

\begin{abstract}
The wild-type Begonia socotrana is rare in cultivation and relatively unknown. However, its pollen is important for producing the widely known and cultivated hybrid begonias. Its biology is outlined in the following.
\end{abstract}

\section{Zusammenfassung}

Die Weihnachts-Begonie (Begonia socotrana) ist eine bislang relativ unbekannte winterblühende Art, die auch nur selten als Wildart in Kultur zu finden ist. Sie ist jedoch ein wichtiger Kreuzungspartner zur Züchtung einiger der bekanntesten Hybridbegonien-Gruppen.

\section{Einleitung}

Viele im Winter blühende Pflanzen werden mit Weihnachten in Verbindung gebracht, darunter auch heimische Arten wie Christrose (Helleborus niger), Laubholz-Misteln (Viscum album) und Stechpalme (Ilex aquifolium). Aber auch Exoten spielen zu dieser Zeit eine besondere Rolle, vor allem wenn sie die klassische weihnachtliche Farbkombination Rot-Grün zeigen. Neben dem bekannten Weihnachtsstern (Euphorbia pulcherrima), Weihnachtskaktus (Schlumbergera) oder dem Ritterstern
(Hippeastrum) spielen die reichblühenden Begonien im Winter auf der Fensterbank eine große Rolle. $\mathrm{Zu}$ diesen gehört auch die Weihnachts-Begonie (Begonia socotrana), eine im Pflanzenhandel weithin unbekannte Art, die aber eine wichtige Rolle als Kreuzungspartner (Pollenspender) für Hybrid-Begonien darstellt. Sie hat die wichtige gartenbauliche Eigenschaft, im Winter zu blühen, worauf sich der Name Weihnachts-Begonie oder Advents-Begonie bezieht. Die reine Wildart gibt es bei uns allerdings nicht zu kaufen.

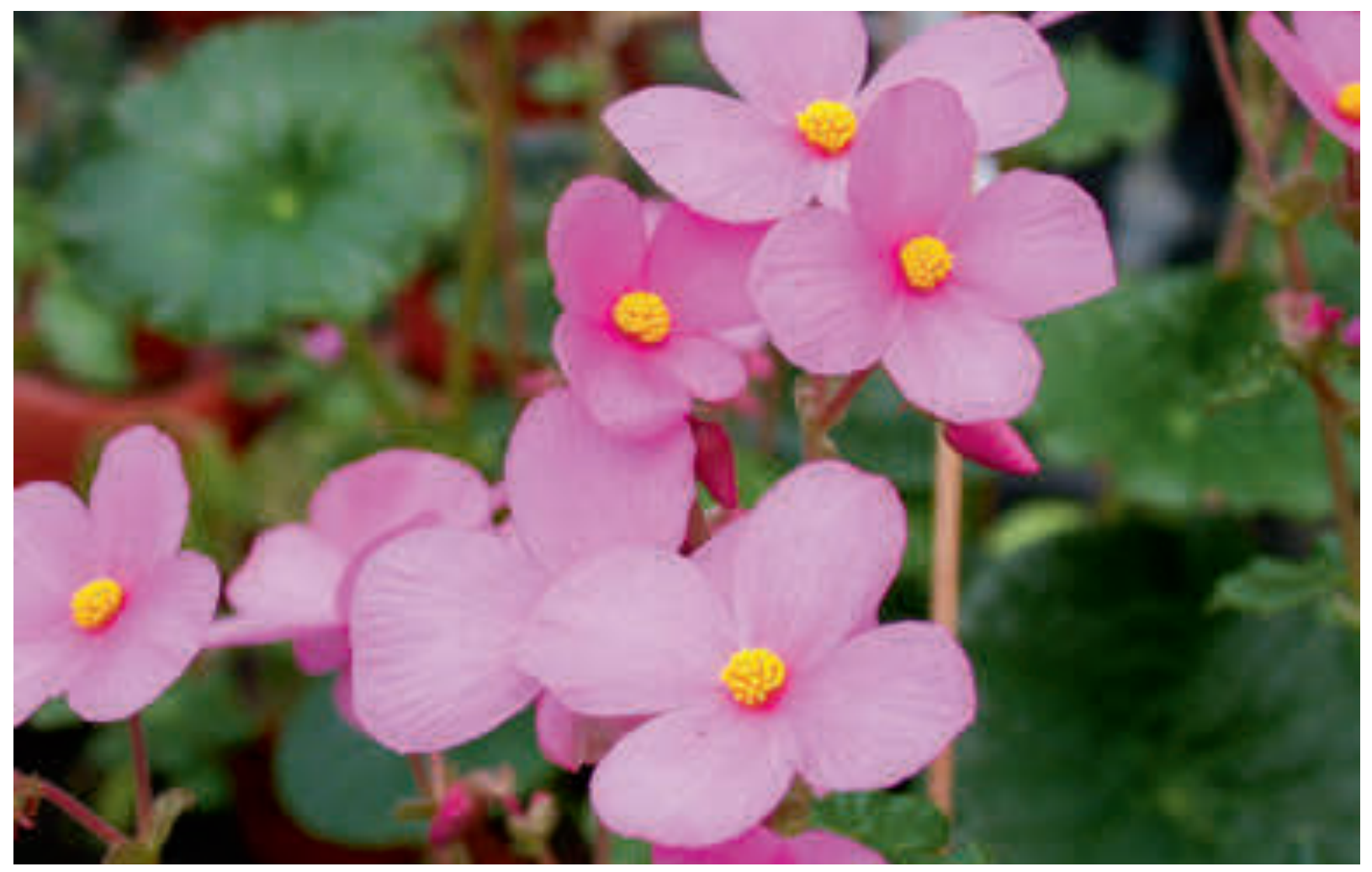




\section{Systematik und Verbreitung}

Die Gattung Begonia ist namensgebend für die Familie der Begoniengewächse (Begoniaceae), die auch als Schiefblattgewächse bekannt ist. Ehemals standen die Begoniaceae in der Ordnung der Begonienartigen (Begoniales). Nach der aktuellen Systematik wird die Familie nun jedoch zu den Kürbisartigen (Cucurbitales) gestellt (Stevens 2001). Die Angaben über die tatsächliche Anzahl von Wildarten schwanken erheblich. Kruse (2000) nennt etwa 900 Arten, MabberLEY (2008) 1400 und Stevens (2001) 1600.

Die Gattung Begonia ist weltweit in den Tropen und Subtropen (außer Australien) heimisch. Die meisten Arten kommen im Unterwuchs feucht-schattiger Wälder vor. Begonia socotrana wurde 1880 auf der Insel Sokotra (östlich vom Horn von Afrika gelegen, Republik Jemen) in einem abgelegenen Bergareal entdeckt. Hier kommt sie häufig in Felsspalten in Kalkgestein vor. Dieser sommertrockene Standort ist für Begonien eher ungewöhnlich.

\section{Morphologie}

\subsection{Habitus}

Begonien sind sehr formenreich, auch hinsichtlich ihrer Wuchsform. So kommen neben der Mehrheit der krautigen Vertreter auch einige Halbsträucher vor. Es gibt kriechende oder rosettig wachsenden Arten sowie kletternde Spreizklimmer. Einige südafrikanische und andine Arten weisen ein kräftiges Rhizom oder knollenartige Verdickungen (Bulbillen) auf (Kruse 2000). Solche bodennahen knolligen Strukturen, die sich am Ende der Regenzeit an der Stammbasis ausbilden, sind auch bei $B$. socotrana vorhanden. Mit diesen Bulbillen übersteht sie die trocken-heißen Sommer an ihrem Naturstandort sehr gut. Zu Beginn der Regenzeit treiben die Bulbillen wieder aus und bilden Spross und Blüten. Im Längsschnitt ist zu er-

Abb. 1 (Seite 150): Blühende Begonia socrotana.

Abb. 2 (oben): Seitenansicht einer Blüte mit kantigem Fruchtknoten.

Abb. 3 (unten): Frisch ausgetriebene Knollen von Begonia socrotana.
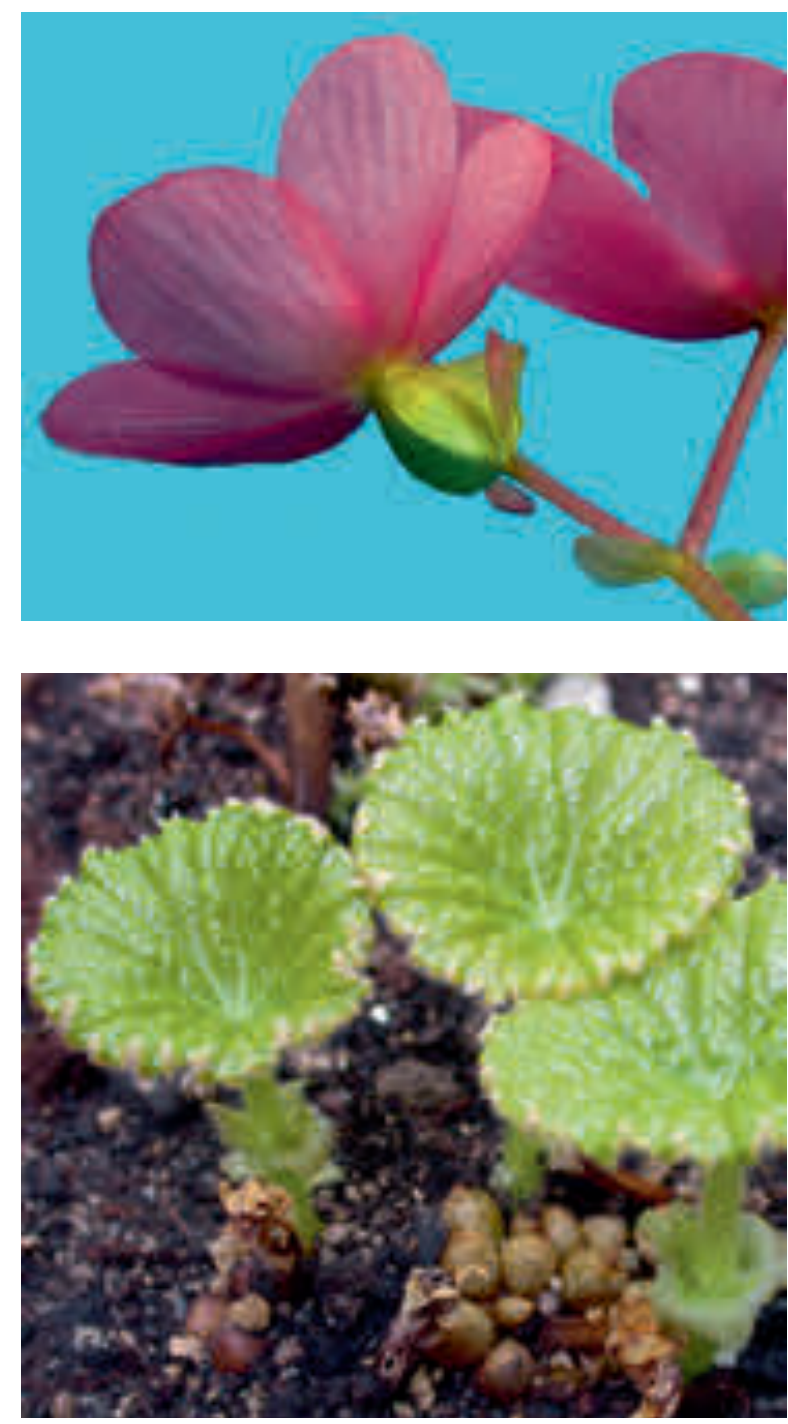

kennen, dass die Bulbillen gestauchte Sprosse sind, die von trocken werdenden Tragblättern schützend umhüllt werden.

\subsection{Blatt}

Die Formen- und Farbenvielfalt der Blätter ist innerhalb der Begonien fast unendlich. Bei den meisten Arten werden stark asymmetrische Blätter hervorgebracht, worauf die deutsche Bezeichnung Schiefblatt zurückzuführen ist. Die Blätter können dunkelgrün bis metallisch glänzend oder auch panaschiert sein, wobei viele Arten auffällige Blattmusterungen aufweisen. Neben kahlen kommen auch samtig behaarte Blätter vor, wie z. B. auch bei der Weihnachts-Begonie. Schildförmige Blätter, wie sie bei der Weihnachts-Begonie im unteren Sprossbereich auftreten, sind innerhalb der Gattung eine Besonderheit. 

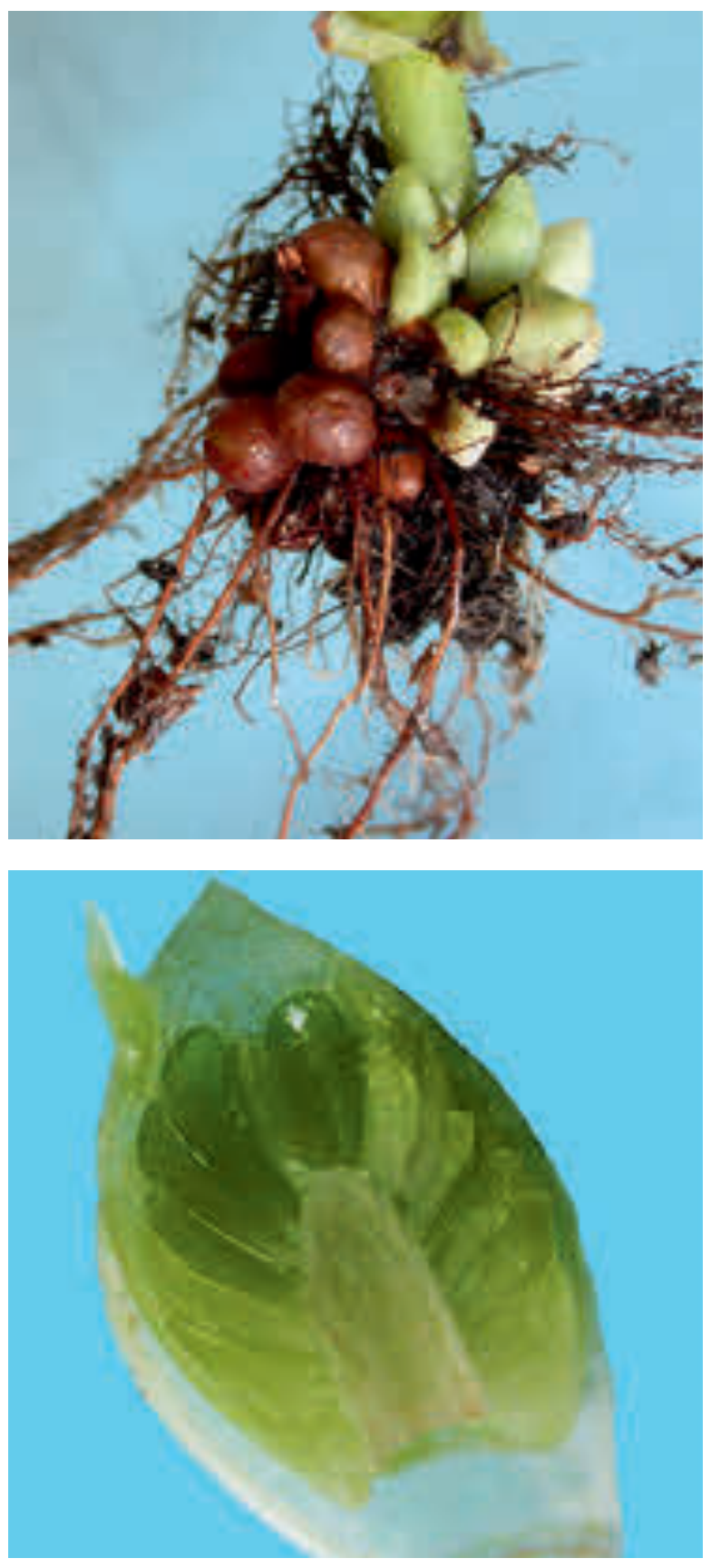

\subsection{Blüte}

Die Blütezeit von Begonia socotrana fällt in die winterliche Regenzeit. Sie ist einhäusig, die männlichen und weiblichen reproduktiven Organe werden in getrenntgeschlechtlichen Blüten hervorgebracht. Zwitterblüten fehlen grundsätzlich. Weibliche Blüten entwickeln 5-6 Blütenblätter, in männlichen sind stets 4 vorhanden. Die Blütenhülle ist undifferenziert, eine Unterteilung in Kelch- und Kronblätter ist nicht möglich. Die Blütenblätter decken sich in der Knospe klappig (Heywood 1982). Männli- che Blüten enthalten zahlreiche Staubblätter. Die Bestäubung wird von Pollen fressenden Insekten vollzogen. Da die weiblichen Blüten keinen Nektar produzieren und demnach für potenzielle Blütenbesucher nicht attraktiv sind, wird mit Hilfe von zwei großen gelben Narbenästen Pollen vorgetäuscht.

Aus dem unterständigen, geflügelten, im Querschnitt mehr oder weniger dreieckigen Fruchtknoten entwickelt sich zur Samenreife eine Kapselfrucht (HeYwood 1982, Leins \& Erbar 2010). Der Fruchtknoten ist aus drei Fruchtblättern verwachsen. Je Fruchtblatt sind zahlreiche Samenanlagen ausgebildet, aus denen sich dann später kleine Samen entwickeln, die nur mit wenig Nährgewebe ausgestattet sind (HeYwood 1982).

\section{Begonien als Zierpflanzen}

Begonien gehören unter den attraktiv blühenden Zierpflanzen zu den beliebtesten Topfpflanzen. Dabei spielen unzählige Hybriden aus der Begonia-Lorraine-Gruppe, die auch im Freien zur Balkon- und Grabbepflanzung geschätzt wird, eine große Rolle. Diese Gruppe ist aus der Kreuzung von $B$. socotrana mit der im südafrikanischen Kapland heimischen $B$. dregei hervorgegangen (KRUSE 2000). Auch innerhalb der Begonia-Elatior-, Begonia-Hiemalis-, und Begonia-Cheimantha-Gruppe nimmt B. socotrana eine bedeutende Rolle als Kreuzungspartner ein.

\section{Literatur}

Mabberley, D. J. 2008: Mabberley's plant book. Cambridge.

Kruse, J. 2000: Begoniales. In: Urania Pflanzenreich, Blütenpflanzen - Berlin.

Heywood, V. H. 1982: Blütenpflanzen der Welt. Stuttgart.

Leins, P. \& Erbar, C. 2010: Flower and fruit. - Stuttgart. Stevens, P. F. 2001: Angiosperm Phylogeny Website, Version 13.

(abzurufen unter http://www.mobot.org/MOBOT/ research/APweb/)

Abb. 4 (oben): Knollen und Wurzeln von Begonia socrotana.

Abb. 5 (unten): Längsschnitt durch eine Knolle von Begonia socrotana. 